\title{
EP-153
}

\section{Breast cancer with asymptomatic metastasis to the gallbladder}

\author{
Hyung Jun KWON*, Sang Geol KIM, Hyun Jeong JEON, Yun Jin HWANG
}

Department of Surgery, Kyungpook National University Chilgok Hospital, Kyungpook National University School of Medicine, Daegu, Korea

Introduction: Breast cancer is the most frequent malignant tumors among female and it is usually associated with metastasis to lungs, bones, liver, and brain. The gallbladder as the site of metastasis from breast carcinoma is extremely rare, and only a few such case reports have been published to date.

Methods: We report the case of a 59-year-old woman diagnosed with breast cancer who underwent cholecystectomy for an incidental finding of gallbladder wall thickening on computed tomography.

Results: A 59-year-old menopausal woman presented with a lump in her right breast for one months. Breast examination revealed an ill-defined palpable area in the right breast. Ultrasonography (US) of the right breast revealed $28 \mathrm{~mm} \times 19 \mathrm{~mm}$ sized irregular hypoechoic mass in the retro-areolar area. This was followed by a US-guided Tru-cut biopsy of the right breast lesion and axillary nodes, which revealed invasive lobular carcinoma. The tumour was positive for oestrogen receptors. Computer tomography showed a wall a thickening in the fundus and body of the gallbladder with multiple gallstones. A single port laparoscopic cholecystectomy was performed, and the postoperative course was uneventful. Histopathology of the gallbladder revealed involvement by an invasive lobular carcinoma.

Conclusions: Metastatic gallbladder involvement is rare, especially in a case of primary breast cancer and It is difficult to suspect a metastatic localization from primary breast cancer in the breast cancer. Even if there is no gastrointestinal symptoms, it is necessary to be well aware of the possibility of metastasis in patients with if imaging reveals gallbladder abnormalities. 